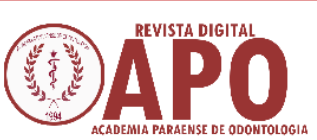

\title{
Modelo tridimensional de estudo da anatomia de molares
}

\section{Three-dimensional study model of the molar anatomy}

\author{
Jeane Moreira Rodrigues ${ }^{1}$ \\ Leda Marques Pena Custódio ${ }^{1}$ \\ Rafael Rodrigues Lima \\ Cláudia Pires Rothbarth ${ }^{2}$
}

\section{RESUMO}

O objetivo desse estudo foi confeccionar modelos tridimensionais do sistema de canais radiculares de molares inferiores diafanizados para o ensino da anatomia dental interna, avaliar a incidência de três, quatro ou mais canais radiculares e classificar segundo Vertucci. Setenta e seis molares inferiores foram submetidos à técnica de diafanização. Após diafanizados, a anatomia dental interna foi examinada, as configurações anatômicas classificadas e os modelos de estudo confeccionados. Dos 76 molares inferiores analisados, 3,9\% possuíam três raízes e 57,9\% possuíam três canais radiculares, $83,8 \%$ das raízes mesiais possuíam dois canais e $67,1 \%$ das raízes distais possuíam um único canal. Nos canais mesiais o tipo IV de Vertucci foi a configuração mais prevalente (47,3\%), seguido do tipo II (24,3\%). Nos canais distais, a maior prevalência foi do tipo I $(67,1 \%)$, seguido do tipo V $(9,2 \%)$. Os modelos tridimensionais de estudo se mostraram uma importante ferramenta para o ensino da anatomia dental interna para alunos de graduação. Os dentes analisados apresentaram uma grande variedade anatômica, mostrando maior prevalência de molares com duas raízes e três canais. Apenas o tipo VIII de Vertucci não foi encontrado.

Palavras chave: Dente molar. Cavidade pulpar. Anatomia.

\begin{abstract}
The study aim was confectioning three-dimensional models of the diaphanized lower molars root canals system for the teaching of the internal dental anatomy, to evaluate the incidence of three, four or more root canals and classifying them according to Vertucci. Seventy-six lower molars underwent diaphanization technique. After being diaphanized, the internal dental anatomy was examined, the anatomical configurations were classified and the study models were confectioned. Among the 76 molars analyzed, $3.9 \%$ had three roots and $57.9 \%$ had three root canals, $83.8 \%$ of the mesial roots had two canals and $67.1 \%$ of the distal roots had a single canal. In the mesial canals, the Vertucci type IV was the most prevalent configuration (47.3\%), followed by the type II (24.3\%). In the distal canals, the most prevalent was the type I $(67.1 \%)$, followed by the type V $(9.2 \%)$. The three-dimensionalstudy models proved to be an important tool for the teaching of the internal dental anatomy to undergraduate students. The analyzed teeth presented a great anatomic variety, showing a higher prevalence of molars with two roots and three canals. Only the type VIII of Vertucci was not found.
\end{abstract}

Keyword: Molar tooth. Dental pulp cavity. Anatomy.

\footnotetext{
${ }^{1}$ Especialista em Endodontia

${ }^{2}$ Professor(a) Doutor(a), Faculdade de Odontologia, Universidade Federal do Pará
} 


\section{INTRODUÇÃO}

O conhecimento da anatomia interna dos canais radiculares é indispensável para o sucesso do tratamento endodôntico, assim o cirurgião-dentista deve compreender as principais características morfológicas do sistema de canais radiculares para o correto planejamento e execução da terapia endodôntica.

Durante as fases iniciais da formação acadêmica dos profissionais de odontologia, a falta de uma visão tridimensional da arquitetura interna dos canais radiculares dificulta a aprendizagem, interfere no entendimento e pode gerar uma deficiência na formação profissional. A inclusão de métodos de estudo da anatomia interna no ensino de graduação, pode contribuir para contornar tais dificuldades.

Um método amplamente utilizado para o estudo da anatomia interna é a diafanização. Esta técnica apresenta-se como um método simples, de fácil execução, rápido, econômico e não necessita de aparelhagens complexas para sua execução. A diafanização revela de forma fiel os detalhes anatômicos o que permite uma visualização tridimensional do elemento dental por transparência, sem alterações significativas da forma original ${ }^{1,2,3}$

Vertucci ${ }^{3}$ utilizou a diafanização para investigar detalhadamente o sistema de canais radiculares de dentes humanos extraídos e estabelecer uma classificação para as diferentes configurações dentais internas encontradas. A partir deste estudo, o autor identificou oito tipos de configurações dentais internas. A classificação proposta por Vertucci ${ }^{3}$ tem servido de parâmetro para outros estudos de anatomia dental interna, além de auxiliar os estudantes na compreensão das configurações anatômicas encontradas.

Uma desvantagem do método da diafanização é a necessidade de armazenamento dos dentes em substância tóxica, dificultando a utilização desse modelo de estudo na rotina diária dos estudantes. Dessa forma, a inclusão dos dentes em blocos de resina transparente, como proposto por Pécora et al. ${ }^{2}$, pode proporcionar o manuseio e facilitar o armazenamento dos dentes diafanizados, permitindo sua utilização segura nas aulas laboratoriais.
Com o propósito de elaborar uma ferramenta pedagógica para o ensino da anatomia dental interna para estudantes de graduação, este trabalho objetivou confeccionar modelos de estudo tridimensionais do sistema de canais radiculares a partir de molares inferiores diafanizados e identificar as diferentes configurações segundo a classificação proposta por Vertucci $^{3}$.

\section{Metodologia}

Este estudo foi aprovado pelo Comitê de Ética em Pesquisa da Universidade Federal do Pará, sob o parecer no 301.984 (CAAE: 16256213.4.0000.0018).

Setenta e seis dentes inferiores, primeiros e segundos molares, extraídos por razões clínicas diversas e doados para pesquisa através do Termo de Consentimento Livre e Esclarecido (TCLE) foram submetidos à técnica de diafanização.

Os espécimes foram limpos removendo-se cálculos, cáries e restaurações. Logo após os dentes foram acessados e desinfetados por imersão em solução de hipoclorito de sódio a $1 \%$, onde permaneceram, em temperatura ambiente, por três dias, com troca da solução desinfetante a cada 24 horas. Em seguida, foram descalcificados em solução de ácido clorídrico a $7 \%$ com troca a cada 24 horas, o que coincidiu com um período de 3 a 7 dias. A descalcificação foi considerada concluída, quando os espécimes apresentaram uma consistência borrachóide associada à coloração branca leitosa e um som surdo ao ser jogado em uma superfície metálica.

Posteriormente à descalcificação, injetou-se uma mistura de gelatina incolor com tinta nanquim na cavidade pulpar até o seu extravasamento pelos ápices radiculares. Em seguida, os dentes foram desidratados em uma bateria crescente de álcool 70\%, 96\% e 100\%, onde permaneceram por 4 horas em cada concentração, após a desidratação os espécimes secaram naturalmente por 24 horas.

Subsequente a esta etapa, os espécimes foram colocados em um recipiente de vidro com tampa contendo salicilato de metila (Polinox, Jundiaí, SP, BR) até apresentarem aspecto transparente. Concluído o processo de diafanização as configurações anatômicas 
dos molares foram observadas com auxílio de um microscópio óptico e classificadas de acordo com Vertucci $^{3}$ (Figura 1).

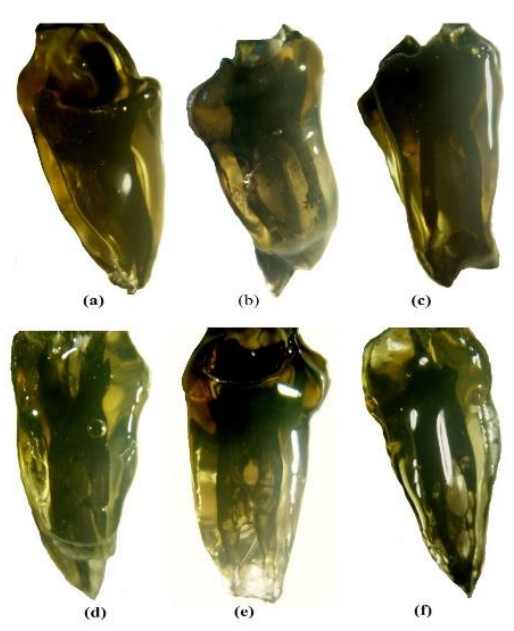

Figura 1 - Configurações observadas neste estudo: (a) tipo I, (b) tipo II, (c) tipo IV, (d) tipo VI, (e) tipo VII e (f) tipo 2-12-1.

Para a obtenção dos modelos de estudo (Figura 2) foi confeccionado um molde a partir de uma cuba plástica para gelo. Uma mistura de $3 \mathrm{ml}$ de salicilato de metila, adicionada a três gotas de catalizador, foi inserida na cuba, onde permaneceu por 14 minutos para que ocorresse a polimerização inicial. Feita a base, um espécime diafanizado foi colocado sobre esta e uma nova camada de salicilato de metila e catalizador foi inserida até cobrir o mesmo, permanecendo por mais 24 horas para a completa polimerização.

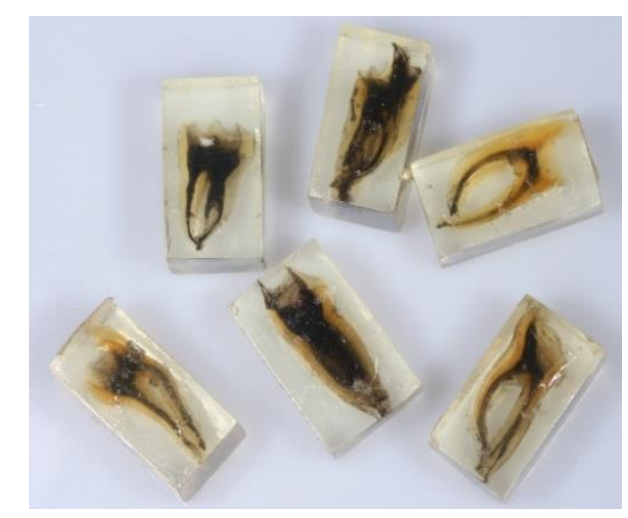

Figura 2 - Molares inferiores incluídos em blocos de resina
O bloco obtido foi então destacado da cuba plástica, recortado com broca Minicut 1512 (Edenta, Suiça) e polido com o auxílio de uma politriz e lixas d'água de granulações 220, 360 e 600 até obter as proporções de $20 \mathrm{~cm}$ de altura, $0,5 \mathrm{~cm}$ largura e $0,5 \mathrm{~cm}$ de profundidade. $\mathrm{O}$ bloco foi então moldado com silicona de condensação (Vigodent, Rio de Janeiro, RJ, BR) para se obter uma matriz padronizada para a confecção dos demais modelos de estudo.

\section{Resultados}

As Tabelas 1 e 2 apresentam as configurações dentais internas da amostra estudada. Dos 76 molares inferiores analisados, 73 (96\%) apresentavam duas raízes e três $(3,9 \%)$ apresentavam uma terceira raiz. Quarenta e quatro $(57,9 \%)$ possuíam três canais radiculares, 24 $(31,6 \%)$ quatro canais, sete $(9,2 \%)$ dois canais e um $(1,4 \%)$ apresentou cinco canais radiculares. Sessenta e duas $(83,8 \%)$ raízes mesiais apresentavam dois canais radiculares e cinquenta e uma $(67,1 \%)$ raízes distais apresentavam apenas um canal radicular.

Tabela 1. Número de raízes e canais observados nos molares inferiores diafanizados.

\begin{tabular}{ccccc}
\hline \multicolumn{5}{c}{ Raízes } \\
Canais & 1 & 2 & 3 & $\%$ \\
\hline 1 & - & - & - & \\
2 & - & $7(9,2)$ & - & $9,2 \%$ \\
3 & - & $42(55,3 \%)$ & $2(2,6 \%)$ & $57,9 \%$ \\
4 & - & $23(30,3 \%)$ & $1(1,3 \%)$ & $31,6 \%$ \\
5 & - & $1(1,3)$ & - & $1,3 \%$ \\
\hline
\end{tabular}

Tanto as raízes mesiais quanto as distais apresentaram grandes variações anatômicas em seus canais radiculares. Nos canais mesiais o tipo IV $(47,3 \%)$ foi a configuração mais encontrada, seguido do tipo II (24,3\%), tipo I (14,9\%), tipo V (6,8\%), tipo III $(2,7 \%)$ e tipo VI $(1,4 \%)$. Duas raízes mesiais apresentaram uma 
configuração dos canais radiculares diferente da proposta por Vertucci ${ }^{3}$, estas foram categorizadas como tipo (2-1-2-1) segundo a classificação de Gulabivala et al. ${ }^{7}$ e tipo (1-3) segundo Sert et al. ${ }^{8}$. Nos canais distais a configuração mais encontrada foi o tipo I $(67,1 \%)$, seguido do tipo V (9,2\%), tipo II (6,6\%), tipo III $(6,6 \%)$, tipo VII $(5,3 \%)$, tipo VI $(3,9 \%)$ e tipo IV $(1,3 \%)$. Os canais mesiolingual, mesiovestibular e distolingual apresentaram configuração tipo I.

Tabela 2. Distribuição dos molares inferiores diafanizados segundo a classificação de Vertucci (1984).

\section{Tipos}

\begin{tabular}{lcccccccc} 
Raízes & I & II & III & IV & V & VI & VII & VIII \\
\hline \multirow{2}{*}{ Raiz Mesial (74) } & 11 & 18 & 2 & 35 & 5 & 1 & - & - \\
& $14,9 \%$ & $24,3 \%$ & $2,7 \%$ & $47,3 \%$ & $6,8 \%$ & $1,4 \%$ & & \\
\hline Raiz Distal (76) & 51 & 5 & 5 & 1 & 7 & 3 & 4 & - \\
& $67,1 \%$ & $6,6 \%$ & $6,6 \%$ & $1,3 \%$ & $9,2 \%$ & $3,9 \%$ & $5,3 \%$ & \\
\hline Mesiolingual (2) & 2 & - & - & - & - & - & - & - \\
Mesiovestibular (2) & 2 & - & - & - & - & - & - & - \\
Distolingual (1) & 1 & - & - & - & - & - & - & - \\
\hline
\end{tabular}

\section{DisCUSSÃo}

A diafanização é uma metodologia bastante eficaz para a análise e compreensão do sistema de canais radiculares, uma vez que proporciona visão anatômica tridimensional. A técnica utilizada neste estudo apresentou-se simples, de fácil execução e baixo custo o que torna seu emprego viável no ensino de graduação.

A visão tridimensional proporcionada pela inclusão do dente diafanizado em blocos de resina (Figura 2) constitui importante ferramenta para o estudo da anatomia dental interna, pois o manuseio dos blocos permite a observação das variações anatômicas em diferentes ângulos.

Estes blocos podem ser utilizados como recurso visual auxiliar no processo de ensino aprendizado, aumentando a curiosidade dos alunos e a velocidade de assimilação de novos conhecimentos ${ }^{4}$.
As configurações anatômicas do sistema de canais radiculares podem estar relacionadas a fatores étnicos variando, portanto, em diferentes populações. Assim a análise da amostra estudada segundo a classificação proposta por Vertucci $^{3}$, permitiu comparar as características e particularidades dos molares estudados, oriundos da população paraense, com aquelas encontradas em estudos realizados em outras localidades.

Neste estudo, a incidência de uma terceira raiz nos molares diafanizados foi de 3,9\% (Tabela 1), similar aos valores encontrados por Al-Qudah e Awawdeh ${ }^{6}$, Peiris et al. ${ }^{11}$ e Ahmed et al. ${ }^{14}$, em populações na Jordânia, Sri Lanka e Sudão, respectivamente.

Esta porcentagem de 3,9\%, no entanto, é menor do que a encontrada por outros autores em diversas localidades ${ }^{5,8,15,21,22,23,24}$. Pablo et al. ${ }^{5}$ realizaram uma análise sistemática de 41 estudos, feitos em diferentes populações, a respeito da anatomia interna e externa de primeiros molares inferiores, seus resultados demonstraram um percentual para terceira raiz de $13 \%$, com maiores prevalências fortemente relacionadas à etnia. Gu et al. ${ }^{15}$, por sua vez, encontraram na população chinesa uma prevalência de $31,9 \%$ de primeiros molares inferiores com três raízes e outros autores também relataram maiores prevalências em populações asiáticas $^{8,15,21,23,24}$.

Apenas três estudos apresentaram valores menores para a incidência de uma terceira raiz que o encontrado na amostra analisada neste estudo, sendo estes realizados em populações iraniana ${ }^{12}$, germânica ${ }^{17} \mathrm{e}$ turca $^{20}$.

Dos artigos que reportaram incidência de molares inferiores com três raízes e identificaram sua localização, esta terceira raiz estava na distal ${ }^{6,7,8,11,12,14,21,23,24}$. Porém dos três espécimes analisados por este estudo, dois apresentaram uma segunda raiz mesial. Esta raiz extra na mesial difere do que é normalmente encontrado, sendo necessário estudos com amostras maiores para se ter valores numéricos mais precisos desta prevalência.

Nos dois dentes que apresentaram duas raízes localizadas na região mesial, estas foram nomeadas como mesiolingual e mesiovestibular, pois eram 
similares, apresentando mesmo tamanho, circunferência, curvatura para distal e classificação tipo I de Vertucci $^{3}$. No dente onde a terceira raiz estava presente na distal, esta era menor que a raiz distal principal, apresentando um percurso diferente e uma curvatura para vestibular, mostrando-se como um apêndice do dente. Esta raiz foi considerada como distolingual e sua classificação foi tipo I de Vertucci ${ }^{3}$.

Dos dentes analisados, foram encontrados $57,9 \%$ de molares inferiores com três canais e $31,6 \%$ com quatro canais (Tabela 1), coincidindo com outros estudos que também indicaram três ser o número mais frequente de canais para este grupo dental ${ }^{6,7,8,12,23}$. Dentre os 76 dentes analisados, apenas um apresentou cinco canais radiculares, sendo representado estatisticamente como $1,3 \%$ da amostra. O baixo percentual para molares inferiores com cinco canais não diferiu dos demais estudos realizados. Pablo et al. ${ }^{4}$ ao realizarem uma análise sistemática observaram que o percentual de molares inferiores com cinco canais foi de 1,4\%.

Observando as raízes mesiais, 83,8\% delas apresentavam dois canais radiculares, estando de acordo com os demais estudos analisados $6,7,11,13,14,19,20,24$. As raízes distais, também seguindo os padrões dos estudos analisados ${ }^{6,7,11,13,14,19,20,24}$, apresentaram uma grande porcentagem de canais únicos $(67,1 \%)$.

Nas raízes mesiais o tipo IV (47,3\%), caracterizado por dois canais separados e distintos estendendo-se da câmara pulpar ao ápice radicular, foi a configuração mais encontrada (Tabela 2), concordando com os demais estudos analisados $6,7,11,13,14,19,20,24$. O tipo II $(24,3 \%)$, onde dois canais saem da câmara pulpar de forma independente e terminam em forame único, foi a segunda configuração mais encontrada, assim como também descrito por outros autores ${ }^{6,7}$. Diferindo do encontrado neste estudo para segunda maior prevalência, dois autores relataram o tipo I, canal único da câmara pulpar até o ápice radicular, como segunda configuração mais encontrada ${ }^{13,20}$ e apenas um relatou ser o tipo $\mathrm{V}$, canal único até o terço médio onde dividese em dois canais e segue assim até o ápice radicular ${ }^{11}$.

Duas raízes mesiais apresentaram classificações diferentes da proposta por Vertucci ${ }^{3}$, estes foram classificados como tipo 2-1-2-1 segundo a classificação de Gulabivala et al. ${ }^{8}$, dois canais saem da câmara pulpar se fundem no terço médio e em seguida se dividem em dois e terminam em forame único no ápice radicular e tipo 1-3 segundo Sert et al. ${ }^{9}$, canal único até o terço médio onde se divide em três canais autônomos que seguem até $\mathrm{o}$ ápice radicular.

Nas raízes distais o Tipo I $(55,3 \%)$ foi a configuração mais encontrada (Tabela 2), estando em conformidade com vários estudos analisados $5,7,11,13,14,19,20,24$. $\mathrm{O}$ tipo $\mathrm{V}$ $(9,2 \%)$ foi a segunda configuração mais encontrada, estando de acordo apenas com Malvar e Corbacho ${ }^{19}$. Outros autores relataram os tipos $\mathrm{II}^{6,7}$ e tipos $\mathrm{IV}^{13,24}$ como segunda configuração mais encontrada.

Nos molares estudados, dentre os oito tipos de canais radiculares propostos na classificação de Vertucci $^{3}$, apenas o tipo VIII, onde três canais distintos originamse na câmara e seguem até o ápice radicular, não foi encontrado.

\section{Conclusão}

Os modelos de estudo demonstraram ser uma importante ferramenta de aprendizagem para os alunos de graduação, visto que proporcionam uma visão tridimensional do sistema de canais radiculares, contribuindo assim para uma melhor compreensão da anatomia dental interna.

Os dentes avaliados apresentaram uma grande variedade anatômica, revelando uma maior prevalência de molares com duas raízes e três canais. Nos molares estudados apenas o tipo VIII da classificação de Vertucci ${ }^{3}$ não foi encontrado, o que sugere que a amostra estudada não difere de forma significativa das demais populações avaliadas em outros estudos sobre anatomia dental interna. 


\section{REFERÊNCIAS}

1. Fachin EVF, Rossi Junior A, Duarte TS. Contribuição ao estudo da técnica da diafanização. Rev Fac Odontol. 1998 jul;39(1):3-8.

2. Pécora JD, Souza Neto MD, Silva RS. Apresentação de uma técnica simplificada de diafanização de dentes e sua inclusão em blocos transparentes. Rev Odonto. 1993; 2(6):384-5.

3. Vertucci FJ. Root canal anatomy of the human permanent teeth. Oral Surg. 1984;58(5):589-99.

4. Alencar CJF, Sequeira E, Wen CL, Haddad AE. Desenvolvimento de objetos de aprendizagem modernos em teleodontologia. ABENO. 2010 jul;10(2):5-11.

5. Pablo OV, Estevez R, Sánchez MP, Heilborn C, Cobenca $\mathrm{N}$. Root anatomy and canal configuration of the permanent mandibular first molar: a systematic review. $J$ Endod. 2010 dec;36(12):1919-31.

6. Al-Qudah AA, Awawdeh LA. Root and canal morphology of mandibular first and second molar teeth in a Jordanian population. Int Endod J. 2009 mar;42:77584.

7. Chourasia HR, Meshram GK, Warhadpande M, Dakshindas D. Root canal morphology of mandibular first permanent molars in an Indian population. Int J Dent. 2012; 6-1.

8. Gulabivala K, Opasanon A, Ng YL, Alavi A. Root and canal morphology of Thai mandibular molars. Int Endod J. 2001 mar;35(1):52-6.

9. Sert S, Aslanalp V, Tanalp J. Investigation of the root canal configurations of mandibular permanent teeth in the Turkish population. Int Endod J. 2004;37:494-9.

10. Cunha FS, Machado G, Neuvald L. Análise da presença do canal cavo-interradicular em molares - estudo in vitro. Rev Odonto Cienc. 2005 mar;20(47):40-4.

11. Peiris R, Takahashi M, Sasaki K, Kanazawa E. Root and canal morphology of permanent mandibular molars in a Sri Lankan population. Ondontol. 2007;95:16-23.

12. Zare MJ, Jafari FG, Mashhadi ME, MoouaviZaher SH, Sarami M. Root and canal morphology of mandibular second molar in an Iranian population by clearing method. J Dent Shiraz Univ Med Scien. 2013;14(2):7881.

13. Rwenyonyi CM, Kutesa A, Muwazi LM, Buwembo W. Root and canal morphology of mandibular first and second permanent molar teeth in a Ugandan population. Odontol. 2009;97:92-6.
14. Ahmed HA, Abu-bakr NH, Yahia NA, Ibrahim YE. Root and canal morphology of permanent mandibular molars in a Sudanese population. Int Endod J. 2007;40:761-6.

15. Gu Y, Lu Q, Wang H, Ding Y, Wang P, Ni L. Root canal morphology of permanent three-rooted mandibular first molars-part I: pulp floor and root canal system. $J$ Endod. 2010 jun;36(6):990-4.

16. Neelakantan P, Subbarao C, Abuja R, Subbarao CV, Gutmann JL. Cone-beam computed tomography study of root and canal morphology of maxillary first and second molars in an Indian population. $J$ Endod. 2010; 36(10):1622-7.

17. Schäfer E, Breuer D, Janzen S. The prevalence of threerooted mandibular permanent first molars in a German population. J Endod. 2009 feb;35(2):202-5.

18. Santos MV. Estudo anatômico da incidência do canal mesiopalatino em primeiros molares superiores com acesso convencional ou através de um desgaste na região de sua embocadura. Cadernos UNIFOA. 2010;13:39-47.

19. Malvar MFG, Corbacho MM. Estudo da anatomia interna de segundos molares inferiores pela técnica de diafanização. J Bras Endod. 2002 dez;3(11):331-7.

20. Nur BG, Ok E, Altunsoy M, Aglarci OS, Colak M, Gungor E. Evaluation of the root and canal morphology of mandibular permanent molars in a South-eastern Turkish population using cone-beam computed tomography. Eur J Dent. 2014 jun;8(2):154-9.

21. Tu MG, Tsai CC, Jou MJ, Chen WL, Chang YF, Chen SY, et al. Prevalence of three-rooted mandibular first molars among Taiwanese individuals. J Endod. 2007; 33(10):1163-6.

22. Garg AK, Tewari RK, Kumar A, Hashmi SH, Agrawal N, Misbra SK. Prevalence of three-rooted mandibular permanent first molars among the Indian population. $J$ Endod. 2010 aug;36(8):1302-6.

23. Zhang R, Wang H, Tian YY, Yu X, Hu T, Dummer PMH. Use of cone-beam computed tomography to evaluate root and canal morphology of mandibular molars in Chinese individuals. Int Endod J. 2011;44:990-9.

24. Chen G, Yao H, Tong C. Investigation of the root canal configuration of mandibular first molars in a Taiwan Chinese population. Int Endod J. 2009 jun;42:1044-9. 